“C 2010 IEEE. Personal use of this material is permitted. Permission from IEEE must be obtained for all other uses, in any current or future media, including reprinting/republishing this material for advertising or promotional purposes, creating new collective works, for resale or redistribution to servers or lists, or reuse of any copyrighted component of this work in other works.” 


\title{
Measurement of Soft Magnetic Composite Material Using an Improved 3D Tester with Flexible Excitation Coils and Novel Sensing Coils
}

\author{
Yongjian $\mathrm{Li}^{1,2}$, Jianguo Zhu' ${ }^{2}$, Senior Member, IEEE, Qingxin Yang ${ }^{1}$, Zhi Wei Lin ${ }^{2}$, \\ Youguang $\mathrm{Guo}^{2}$, Senior Member, IEEE, and Yi Wang ${ }^{2}$ \\ ${ }^{1}$ Province-Ministry Joint Key Laboratory of Electromagnetic Field and Electrical Apparatus Reliability, \\ Hebei University of Technology, Tianjin, 300130, China \\ ${ }^{2}$ School of Electrical, Mechanical and Mechatronic Systems, University of Technology, Syd ney, NSW 2007, Australia
}

In this paper, accurate measurement of three dimensional (3D) magnetic properties of soft magnetic composite (SMC) material is carried out by using an improved 3D tester with adjustable excitation coils and novel sensing coils attached upon the surface of the SMC specimen. Comparing with the conventional 3D tester operating at $50 \mathrm{~Hz}$, the improved 3D tester enables measurements over wide frequency range from $2 \mathrm{~Hz}$ to $1000 \mathrm{~Hz}$. The relationship between the $\mathrm{B}$ vector and $\mathrm{H}$ vector are measured under both alternating and rotating flux conditions, and the core loss features are analyzed. These experimental results are crucial for designing new $\mathrm{SMC}$ electrical machines, which are expected to operate at $200 \mathrm{~Hz}$ or above.

Index Terms - Flexible excitation coil, improved 3D magnetic tester, sensing coil, soft magnetic composite (SMC).

\section{INTRODUCTION}

$\mathrm{T}$ HREE DIMENSIONAL (3D) magnetic properties of the soft magnetic composite (SMC) material are important for analyzing the operational performance of electrical machines [1], in which the magnetic flux density in SMC is rotational. The SMC materials display complicated relationship between magnetic field strength vector $\mathbf{H}$ and magnetic flux density vector $\mathbf{B}$ under $3 \mathrm{D}$ excitation due to the magnetic domain wall dynamics and non-uniform distribution of local magnetization [2]. Magnetic properties of SMC materials over wide frequency range are crucial factors for accurate analysis of magnetic field in 3D flux machines [3]. By using a 3D magnetic property tester developed at University of Technology, Sydney (UTS), a kind of SMC material, SOMALOYTM500 developed by Höganäs AB, Sweden, has been measured and analyzed under $50 \mathrm{~Hz}$ excitation [4]-[8]. However, there are no studies on 3D magnetic measurement over wide range of excitation frequency, which is strongly demanded by electrical machine designers. In this paper, an improved 3D tester with adjustable multi-layer excitation coils is discussed, in the centre of which novel precision $B-H$ sensing coils are attached on a cubic SMC specimen. Various hysteresis loops and core losses at different frequencies under alternating excitation are presented and analyzed. Additionally, 3D B-H loci and corresponding core losses are also analyzed in detail when the $\mathbf{B}$ vector is controlled to be ellipses evolving from a straight line into a circle in three orthogonal planes.

\section{IMPROVED 3D MAGNETIC TEST ING SYSTEM}

\section{A. Structure of Improved Excitation Coil}

The first 3D magnetic property tester was set up to measure 3D hysteresis loci and losses at $50 \mathrm{~Hz}$ [5]. In order to measure properties over wide range of frequency the original excitation coil was reformed to cover three ranges of frequency, $2 \mathrm{~Hz}-20$ $\mathrm{Hz}, 20 \mathrm{~Hz}-200 \mathrm{~Hz}$, and $200 \mathrm{~Hz}-1000 \mathrm{~Hz}$. Each new coil is consisted of three coils. Fig. 1 shows the structure of improved multi-layer excitation coil. The first two layers, 70 turns, are designed for high frequency range. The middle six layers combining with the first two layers are for medium frequency range. The outer eight layers together with inner eight layers are for low frequency range.

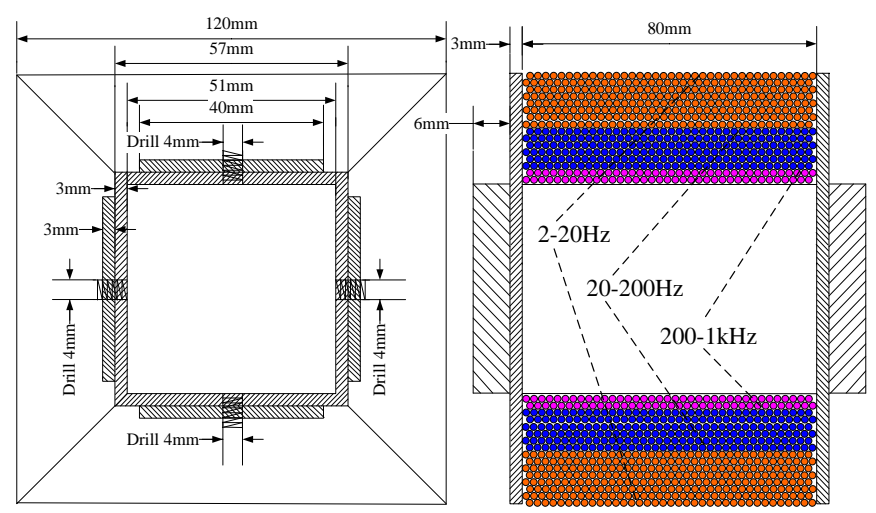

Fig. 1. Scheme of the improved excitation coil.

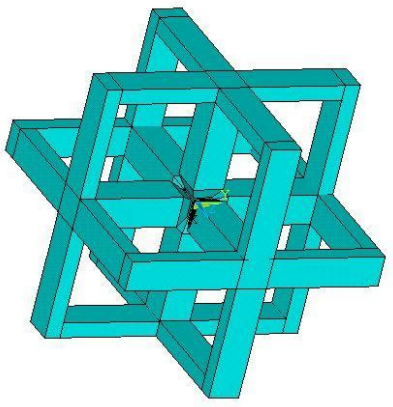

(a)

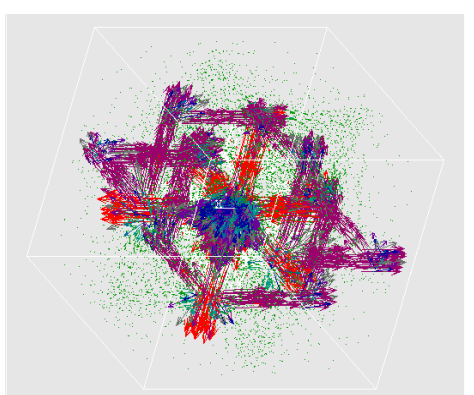

(b)
Fig. 2. Numerical analysis of 3D tester: (a) structure of the 3D tester, (b) magnet ic field distribution corresponding to $3 \mathrm{D}$ field excitation. 
TABLE I

COEFFICIENTS OF THE $H$ AND $B$ SENSING COILS (UNIT: $\mathrm{m}^{2}$ )

\begin{tabular}{cccccccccccc}
\hline \hline$K_{H x}$ & $K_{H y}$ & $K_{H z}$ & $K_{B x}$ & $K_{B y}$ & $K_{B z}$ & $K_{H x y}$ & $K_{H x z}$ & $K_{H y x}$ & $K_{H y z}$ & $K_{H z x}$ & $K_{H z y}$ \\
\hline $2.17 \times 10^{-3}$ & $2.22 \times 10^{-3}$ & $2.16 \times 10^{-3}$ & $1.89 \times 10^{-3}$ & $1.80 \times 10^{-3}$ & $1.80 \times 10^{-3}$ & $8.82 \times 10^{-7}$ & $3.69 \times 10^{-5}$ & $3.22 \times 10^{-5}$ & $7.41 \times 10^{-7}$ & $1.25 \times 10^{-6}$ & $6.16 \times 10^{-5}$ \\
\hline \hline
\end{tabular}

In order to validate the excitation coil structure, 3D finite element magnetic field analysis has been performed to exhibit the flux distribution in the Hi-B lamination yoke and SMC specimen. Fig. 2 shows the tester structure and magnetic field distribution under $3 \mathrm{D}$ excitation when three pairs of excitation coils in three orthogonal axes are connected in series. The simulation result shows that generated fields are uniform and the field can be up to $1.9 \mathrm{~T}$ in the center of 3D tester.

\section{B. Improved Structure of the Sensing Coils}

The original sensing box was composed of six cross-type $H$ coils attached on six surfaces of a cubic specimen. Three $B$ coils was wound around three axes of the cubic specimen [5]. The size of the $H$ coil is approximate equal to that of the specimen side surface. Therefore, the magnetic field at the measured area is not uniform since the demagnetization factor. The improved sensing box combines six $H$ coils and six $B$ coils, as shown in Fig. 3. The small circle $B$ coil is embedded in the center of the epoxy resin frame, around which $H$ coil is wound and covers the $B$ coil. A double-layer winding structure is adopted to eliminate electromotive force (emf) induced by unwanted stray field, as shown in Fig. 3(a). Be noted that the two terminals of each coil are twisted to eliminate interference from stray field. The six $B-H$ sensing coils are integrated to a sensing box and all coils are located at the center of each surface. As a result, a cubic specimen can be easily mounted into the sensing box and the surface of specimen is as close as possible to the $B-H$ sensing coils, as shown in Fig. 3(b). Also the measured field is uniform and close to the fields in specimen.

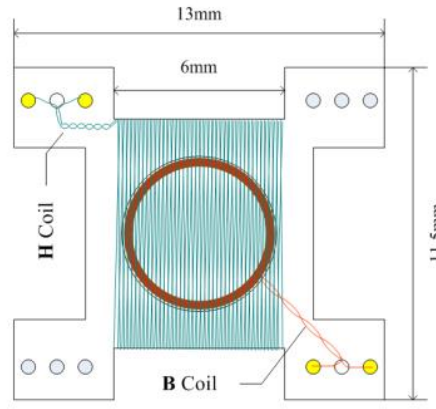

(a)

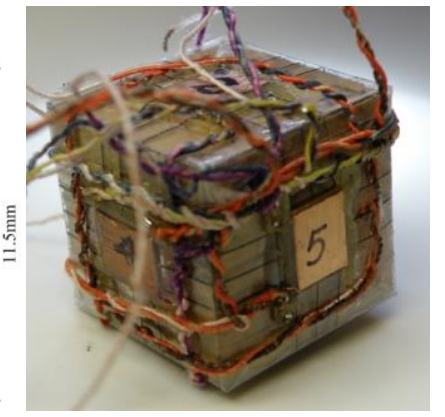

(b)
Fig. 3. Structure of the improved sensing coils: (a) schematic $B-H$ coil, (b) cubic specimen with sensing coils.

\section{Calibration ofthe Sensing Coils}

For accurate measurement of 3D magnetic properties, the sensing box was calibrated in a long solenoid to obtain box coefficients [9], $K$.

$$
K=\frac{E}{\sqrt{2} \pi f \mu_{0} H_{m}}
$$

where $E$ is the rms value of the induced $e m f, \mu_{0} H_{\mathrm{m}}$ is the peak value of the flux density in the center of the solenoid, $f$ is the excitation frequency.

The coefficients are used to calculate $\mathbf{B}$ and $\mathbf{H}$ vectors in the specimen as shown in (2), (3). The average $K$ values are shown in Table I. The off-diagonal coefficients for $B$ coils are ignored since they are very small comparing with diagonal values. Hence, the $\mathbf{B}$ vector can be simply calculated from (2). However, for $H$ coils, the off-diagonal elements cannot be ignored because the field perpendicular to the laminar $H$ coil also induced large emf due to irregularity of the $H$ coil. According to Faraday Induction Law, the inducted voltages crossing $H$ and $B$ coils can be expressed as

$$
\begin{aligned}
& \left\{\begin{array}{l}
U_{B x}=K_{B z} \frac{\mathrm{d} B_{z}}{\mathrm{~d} t} \\
U_{B y}=K_{B x} \frac{\mathrm{d} B_{x}}{\mathrm{~d} t} \\
U_{B z}=K_{B y} \frac{\mathrm{d} B_{y}}{\mathrm{~d} t}
\end{array}\right. \\
& \left.\left\{\begin{array}{l}
U_{H x}=\mu_{0}\left(K_{H x x} \frac{\mathrm{d} H_{x}}{\mathrm{~d} t}+K_{H x y} \frac{\mathrm{d} H_{y}}{\mathrm{~d} t}+\frac{K_{H x z}}{\mu_{0}} \frac{\mathrm{d} B_{z}}{\mathrm{~d} t}\right) \\
U_{H z}=\mu_{0}\left(K_{H y x} \frac{\mathrm{d} B_{x}}{\mathrm{~d} t}+K_{H y y} \frac{\mathrm{d} H_{y}}{\mathrm{~d} t}+K_{H y z} \frac{\mathrm{d} H_{z}}{\mathrm{~d} t}\right) \\
\mathrm{d} t
\end{array}\right\} \frac{K_{H z y}}{\mu_{0}} \frac{\mathrm{d} B_{y}}{\mathrm{~d} t}+K_{H z z} \frac{\mathrm{d} H_{z}}{\mathrm{~d} t}\right)
\end{aligned}
$$

The magnetic field strength and flux density components can then be worked out.

\section{EXPERIMENT AL MEASUREMENT}

By using the improved 3D tester, experiments in wide ranges of excitation frequency have been carried out under both alternating and rotating magnetization conditions.

\section{A. Alternating Magnetic Properties}

Fig. 4 shows a group of $\mathbf{B}-\mathbf{H}$ hysteres is loops at different flux densities at $5 \mathrm{~Hz}, 20 \mathrm{~Hz}, 500 \mathrm{~Hz}$, and $1000 \mathrm{~Hz}$. It can be found out that the maximum flux density at $1000 \mathrm{~Hz}$ is improved to $0.54 \mathrm{~T}$, compared with $0.1 \mathrm{~T}$ of original tester. Fig. 5 shows the loops at $50 \mathrm{~Hz}$ along $x$-, $y$-, and $z$-axes. As shown, the loops for the $x$ - and $z$-axis are similar, while the $y$ axis seems to be the easy axis, though the specimen is expected to be magnetically isotropic. This phenomenon is also observed at other frequencies. It may imply that the particles of the SMC specimen are much closer along the $y$ direction than along the $x$ - and $z$-directions, that is, higher mass density and weaker demagnetization field along the $y$ direction [10]. Fig. 6 plots core losses along $y$-axis at different frequencies. It is noted that the core loss increases quickly at high frequency range. Therefore, for SMC electrical machine the flux density in the SMC core should be chosen lower than 
$0.5 \mathrm{~T}$ to reduce the total core loss [6].

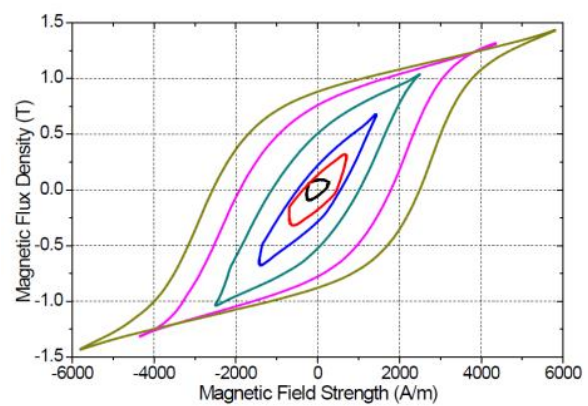

(a)

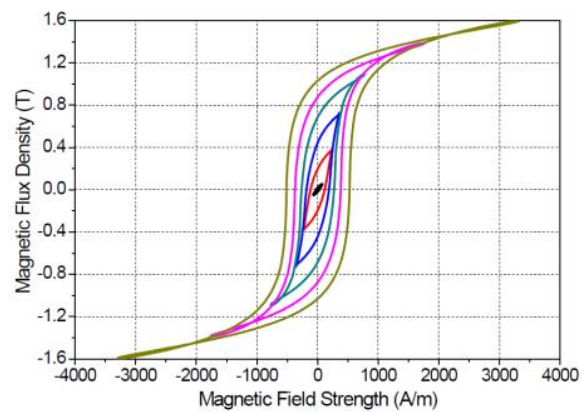

(b)

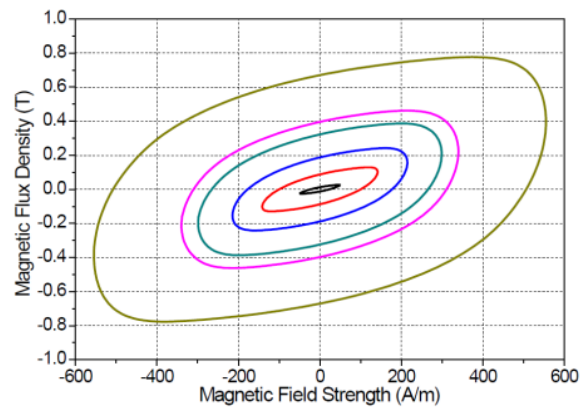

(c)

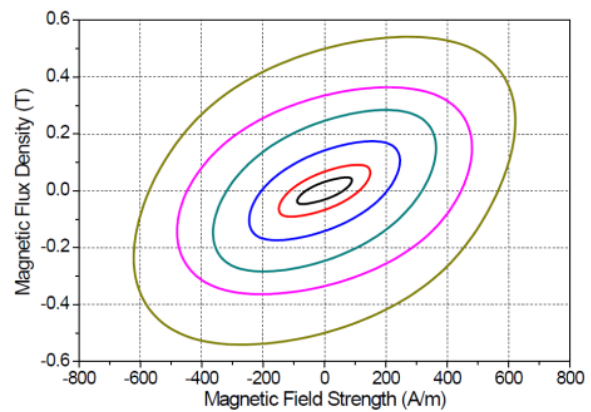

(d)

Fig. 4. Hysteresis loops in alternating magnetic field excitations: (a) $\mathbf{B}-\mathbf{H}$ loops at $5 \mathrm{~Hz}$ on the $x$-axis, (b) B-H loops at $20 \mathrm{~Hz}$ on the $y$-axis, (c) B-H loops at $500 \mathrm{~Hz}$ on the $y$-axis, (d) $\mathbf{B}$-H loops at $1000 \mathrm{~Hz}$ on the $y$-axis.

\section{B. 3-D Magnetic Properties}

The rotational B-H properties are also investigated. The controlled circular $\mathbf{B}$ loci and the corresponding $\mathbf{H}$ loci are measured at $50 \mathrm{~Hz}$ with increasing $\mathbf{B}$ amplitude (up to $1.3 \mathrm{~T}$ ) in the xoy-, yoz- and zox-planes, as shown in Fig. 7. It is observed that the $\mathbf{B}$ and $\mathbf{H}$ loci lie in the same magnetization plane. The $\mathbf{H}$ loci in the xoy- and yoz-planes evolve from ellipses into rectangular loops while the $\mathbf{H}$ loci in the zox- plane changes from circles into square loops. This result is consistent with alternating hysteresis loops as shown in Fig. 5, due to easy magnetization along the $y$-axis.

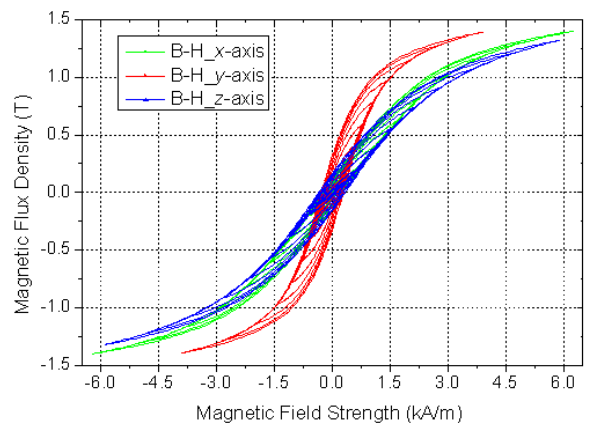

Fig. 5. Hysteresis loops at $50 \mathrm{~Hz}$ on $x$-, $y$-and $z$-axes.

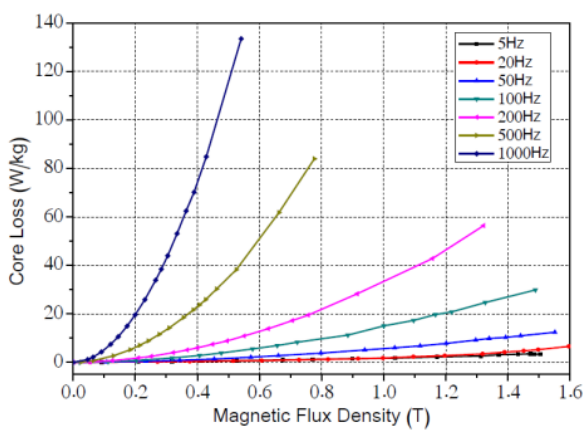

Fig. 6. Core losses at different frequencies on $y$-axis.

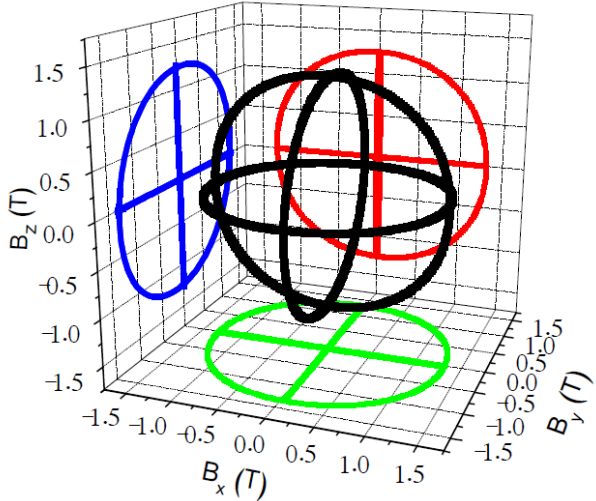

(a)

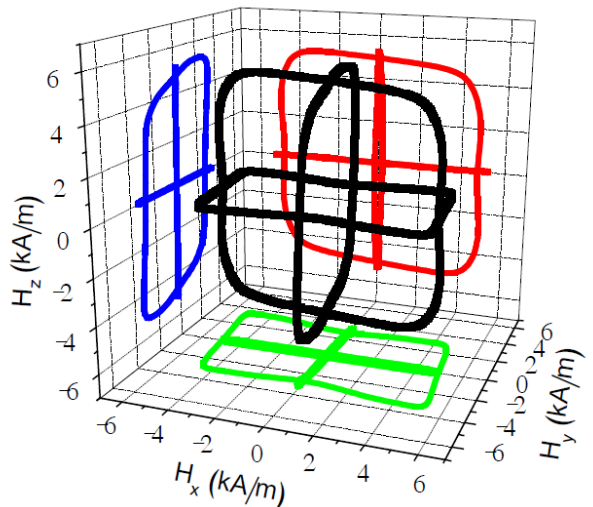

(b)

Fig. 7. Round $\mathbf{B}$ loci and corresponding $\mathbf{H}$ loci in $x o y$-, yoz-, zox-planes and projections in three planes, $B_{x}=B_{y}=B_{z}=1.3 \mathrm{~T}$ : (a) $\mathbf{B}$ loci and corresponding projections, (b) $\mathbf{H}$ loci and corresponding projections. 


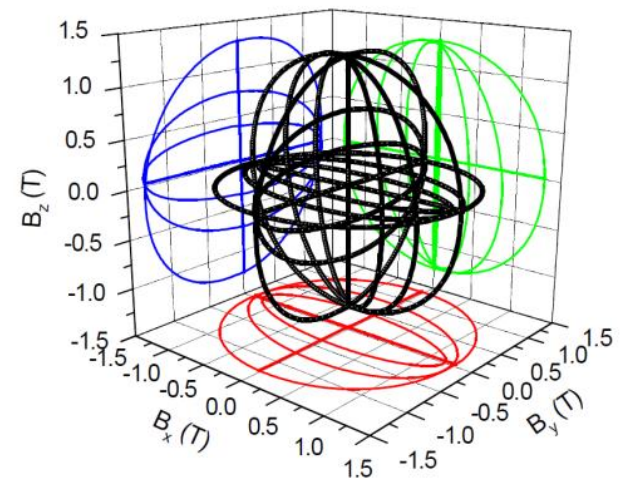

(a)

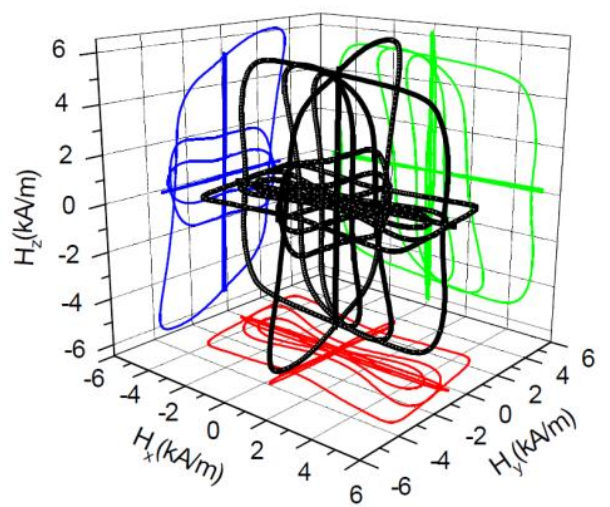

(b)

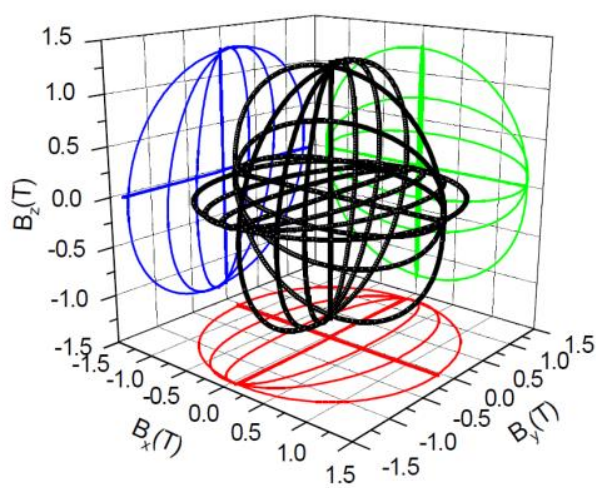

(c)

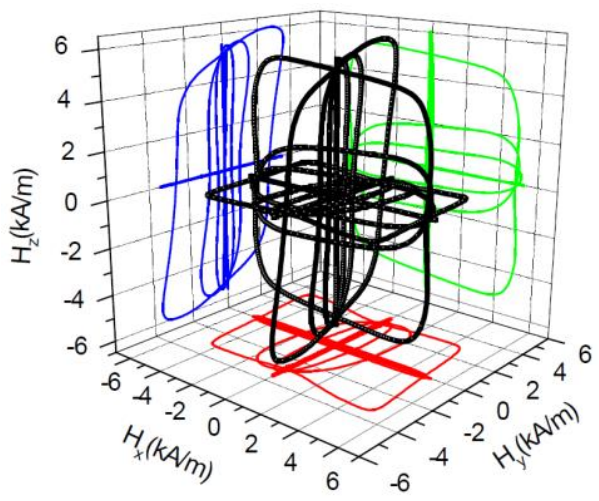

(d)

Fig. 8. 3D magnetic properties of the SMC specimen with elliptical rotating flux density vector at $200 \mathrm{~Hz}$ : (a) elliptical $\mathbf{B}$ loci (the axis ratio $\varepsilon$ is controled from 0 to 1 ) and projections in $x o y$-, yoz-, zox-plane, and the major axis is $x, y$, and $z$ respectively, (b) $\mathbf{H}$ loci and projections corresponding to (a) in three planes, (c) elliptical B loci and projections in $x o y$-, yoz-, zox-plane, and the major axis is $y, z$, and $x$ respectively, (d) $\mathbf{H}$ loci and projections corresponding to (c) in three planes.
The rotational loci at low frequency are similar with that at $200 \mathrm{~Hz}$, but at high frequency range loci are distorted due to higher-order harmonics. The $\mathbf{H}$ loci change to irregular shapes with increasing excitation frequency. Fig. 8 illustrates the B and $\mathbf{H}$ loci at $200 \mathrm{~Hz}$ when the $\mathbf{B}$ loci are controlled to be a series of ellipses, where the axis ratio, $\varepsilon$, of the minor axis to the major axis changes from 0 to 1 . Compared with the $\mathbf{H}$ locus at $50 \mathrm{~Hz}$, as shown in Fig. 7(b), the $\mathbf{H}$ locus obtained at $200 \mathrm{~Hz}$ is slightly different when $\mathbf{B}$ locus are also controlled to be a circle $(\varepsilon=1)$ though they are expected to be the same.

\section{CONCLUSION}

The 3D magnetic property tester is reformed to expand measurement frequency up to $1000 \mathrm{~Hz}$ by using flexible mult ilayer excitation coils. In addition, new designed sensing box can measure $\mathbf{B}$ and $\mathbf{H}$ vector in a cubic specimen more accurately and has a flexible structure to change the specimen. The results show that $\mathbf{H}$ loci in a cubic SMC specimen are different at $50 \mathrm{~Hz}$ and $200 \mathrm{~Hz}$ when $\mathbf{B}$ loci are controlled to be the same probably due to higher-order harmonics. The experimental data provide valuable reference to design and optimize the 3D flux electrical machines.

\section{ACKNOWLEDGMENT}

This work is supported in part by the China Hebei Provincial Natural Science Foundation under Grant No. E2008000051.

\section{REFERENCES}

[1] P. Oxley, "Apparatus for Magnetization and Efficient Demagnetization of Soft Magnetic Materials," IEEE Trans. Magn., vol. 45, no. 9, pp. 3274-3283, 2009.

[2] V. Basso and G. Bertotti, "Hysteresis in soft magnetic materials," $J$. Magn. Magn. Mater., vol. 215,pp. 1-5, 2000.

[3] Y. G. Guo, J. G. Zhu, and D. G. Dorrell, "Design and Analysis of a Claw Pole Permanent Magnet Motor With Molded Soft Magnetic Composite Core," IEEE Trans. Magn., vol. 45, no. 10, pp. 4582-4585, 2009.

[4] Soft magnetic composites from Höganäs Metal Powders-SOMALOY ${ }^{\mathrm{TM}}$ 500, Höganäs Product Manual, 1997.

[5] J. G. Zhu, J. J. Zhong, Z. W. Lin, and J. D. Sievert, "Measurement of magnetic properties under 3-D magnetic excitations," IEEE Trans. Magn., vol. 39, no. 5, pp. 3429-3431, 2003.

[6] Y. G. Guo, J. G. Zhu, P. A. Watterson, and W. Wu, "Comparative study of 3D flux electrical machines with soft magnetic composite core," IEEE Trans. Ind. Appl., vol. 39, no. 6, pp. 1696-1703, 2003.

[7] J. J. Zhong, Y. G. Guo, J. G. Zhu, and Z. W. Lin, "Characteristics of soft magnetic composite material under rotating magnetic fluxes," J. Magn. Magn. Mater., vol. 299, pp. 29-34, 2006.

[8] Z. W. Lin and J. G. Zhu, "Three-dimensional magnet ic properties of soft magnetic composite materials," J. Magn. Magn. Mater., vol. 312, pp. 158-163, 2007

[9] Y. G. Guo, J. G. Zhu, Z. W. Lin, J. J. Zhong, H. Y. Lu, and S. H. Wang, "Calibration of Sensing Coils of a Three-Dimensional Magnetic Property Tester,"IEEE Trans. Magn., vol.42,pp. 3243-3245, 2006.

[10] Z. W. Lin, J. G. Zhu, and Y.G. Guo, "Three-dimensional hysteresis of soft magnetic composite," J. Appl. Phys., vol. 99, 08D909, 2006. 